\title{
On hydromagnetic waves of finite amplitude in a cold plasma
}

\author{
By P. G. SAFFMAN \\ King's College, London
}

(Received 21 April 1961)

The existence of steady, one-dimensional, finite-amplitude waves in a cold collision-free plasma is investigated for the case in which the plasma and the magnetic field are uniform far ahead of the wave. It is supposed that the magnetic field at infinity is inclined at an angle $\beta$ to the direction normal to the plane of the waves $\left(0 \leqslant \beta \leqslant \frac{1}{2} \pi\right)$. It is shown that the problem is equivalent to determining the orbits of a particle in a uniformly rotating field of force.

Two types of waves are found to exist for $\beta \neq 0$ : solitary waves and quasishocks. In a quasi-shock, the plasma and magnetic field oscillate irregularly behind the wave front about mean values, which are different from the values ahead of the wave. The width of the wave front is of the order of the ion gyroradius. The quasi-shocks resemble oblique magnetohydrodynamic shock-waves. For the case $\beta=0$, only solitary waves exist, which have already been described (Saffman 1961).

The stability of the waves is considered, and it is concluded that the quasishocks are stable but that the solitary waves for $\beta \neq 0$ are unstable.

\section{Introduction}

The existence of steady one-dimensional hydromagnetic solitary waves of finite amplitude in a cold plasma has been demonstrated by Adlam \& Allen (1958), Davis, Lüst \& Schlüter (1958), and the author (1961). The two former sets of authors examined the transverse case in which the magnetic field lies in the plane of the wave and is perpendicular to the direction in which the wave propagates. The present author considered the longitudinal case in which the magnetic field ahead of and behind the wave is parallel to the direction of propagation and perpendicular to the plane of the wave.

An obvious extension of this work is to consider the case in which the magnetic field is inclined at an acute angle to the plane of the wave. In the present paper, we shall set up the equations for such waves and discuss the general nature of their solutions. It is found that the problem is equivalent to determining the orbits of a particle in a uniformly rotating field of force. (The motion is also analogous to that of a particle in a smooth spinning bowl of a particular shape.) The equations are non-linear, and it has not proved possible to find exact solutions. However, general properties of the orbits can be obtained by using general theorems for the motion of dynamical systems.

It transpires that there is a fundamental difference between the oblique waves 
and the longitudinal ones. Whereas, in the latter case, the equations only admit of a unique solution, there may be infinitely many solutions for oblique waves (and also for the transverse case with $\beta=\frac{1}{2} \pi$ ). Moreover, there are strong indications (although rigorous proofs are lacking) that some of the solutions represent solitary waves, and that the others describe waves or oscillations of semi-infinite extent. That is, there exist solutions in which the plasma is uniform ahead of a wave front, and its properties vary with distance behind the front in an oscillatory but non-periodic fashion. It is possible to calculate the mean values of the velocities and of the magnetic field behind the wave front (or at least reduce the problem to quadrature), and since the change is essentially irreversible we have quantitative information about a flow which resembles an oblique collision-free magnetohydrodynamic shock-wave.

It is also shown in $\$ 8$ that none of the oblique solitary waves (including the transverse case but excluding the longitudinal one) are likely to be physically significant because they are all unstable.

\section{The equations of motion}

We shall now set up the equations describing steady one-dimensional hydromagnetic waves in a cold plasma. A frame of reference moving with the waves is chosen, so that all variables are functions of only one space co-ordinate, $x$ say. In the present work, we shall only consider the case in which the plasma and the magnetic field ahead of the waves (at $x=-\infty$ ) are uniform. There are then three possibilities for any waves which we may obtain. They may be solitary waves in which conditions behind the wave (at $x=+\infty$ ) are uniform and the same as those ahead of the wave; or true shocks in which conditions at $x=+\infty$ are uniform but different from those at $x=-\infty$; or waves of semi-infinite extent in which the plasma behind the wave does not return to a uniform state but the variables oscillate with $x$ in some manner. This last possibility we shall term a quasi-shock.

We denote by $U$ the component of the velocity of the ions and electrons in the $x$-direction (i.e. normal to the plane of the wave) at infinity ahead of the wave, and the number density of the ions and electrons at $x=-\infty$ by $N$.

We suppose that the magnetic field ahead of the wave is of strength $H_{0}$ and inclined at an angle $\beta$ to the $x$-axis. Moreover, without loss of generality we may superpose any velocity in the $y$-or $z$-directions (i.e. in the plane of the wave), and we do this so that the electric field ahead of the wave is zero. This implies that the velocity and magnetic field are parallel (see figure 1).

The equations of motion can be made non-dimensional (as in Saffman 1961) by normalizing the variables with respect to the velocity $U$, the number density $N$, the magnetic intensity $H_{0}$, and the length

$$
l=a c \sqrt{ }\left(m_{i} m_{e}\right) / e H_{0} .
$$

In (2.1), $a$ is the Alfvén velocity $H_{0} /\left\{4 \pi N\left(m_{i}+m_{e}\right)\right\}^{\frac{1}{2}}, c$ is the velocity of light, $e$ is the electronic charge, and $m_{i}, m_{e}$ are the masses of the ions and electrons, respectively. Gaussian units are employed.

We make the usual quasi-neutral approximation, appropriate to hydromagnetic waves, that the number densities of ions and electrons are equal, 
from which it follows by the equation of continuity that the $x$-components of the ion and electron velocities are equal. Some immediate deductions from Maxwell's equations for the electromagnetic field can also be made. They are that the $x$-component of the magnetic field is constant, and that only the $x$-component of the electric field is non-zero. Then the dimensionless magnetic field is $\mathbf{h}=\left(\cos \beta, h_{2}, h_{3}\right)$ and the dimensionless electric field is $\mathbf{E}=(E, 0,0)$. The dimensionless velocity of the ions is $\mathbf{u}_{i}=\left(u, v_{i}, w_{i}\right)$ and that of the electrons is $\mathbf{u}_{e}=\left(u, v_{e}, w_{e}\right)$.

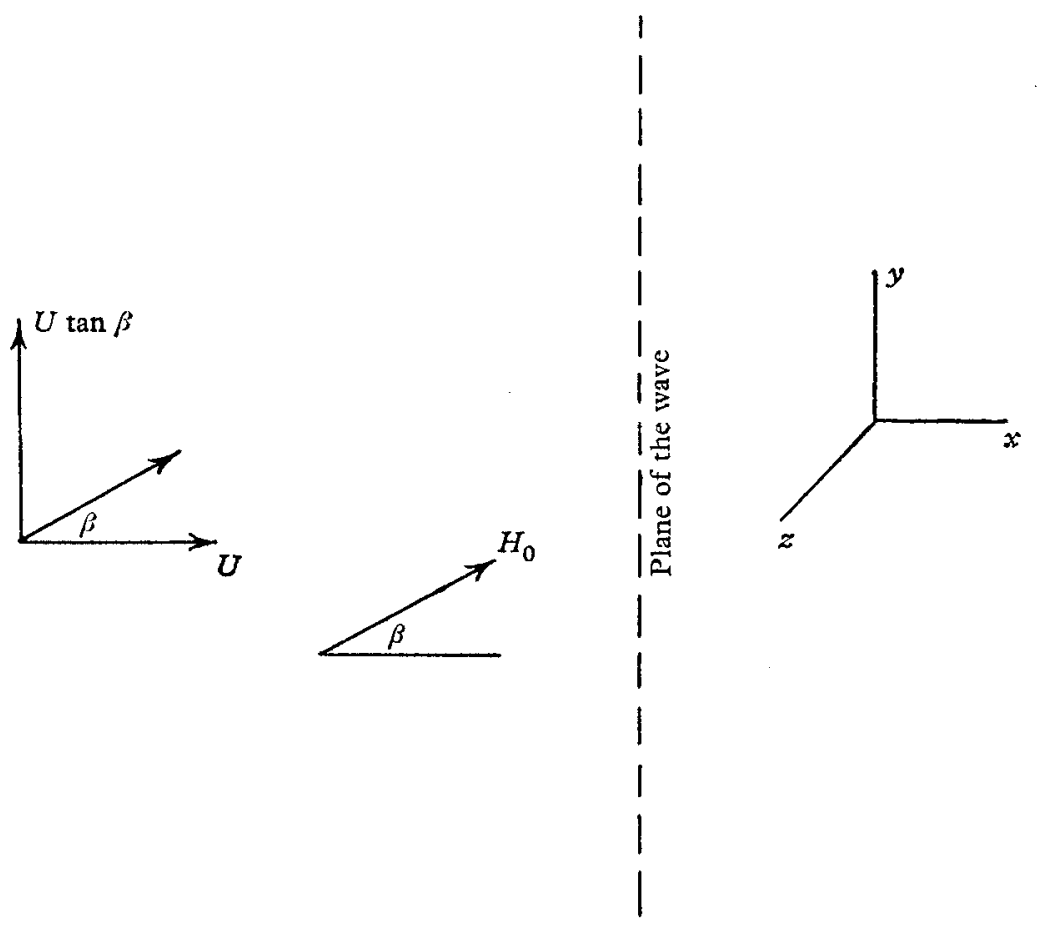

Fraure 1. Sketch of the geometry.

The (dimensionless) equations of motion for these variables take the form (see Montgomery 1959; or Saffman 1961)

and

$$
\begin{aligned}
\frac{M u}{\gamma} \frac{d \mathbf{u}_{i}}{d x} & =\mathbf{E}+\mathbf{u}_{i} \wedge \mathbf{h}, \\
M \gamma u \frac{d \mathbf{u}_{e}}{d x} & =-\mathbf{E}-\mathbf{u}_{e^{\wedge}} \mathbf{h}, \\
u \operatorname{curl} \mathbf{h} & =\frac{M}{\gamma+\gamma^{-1}}\left(\mathbf{u}_{i}-\mathbf{u}_{e}\right) .
\end{aligned}
$$

Here $\gamma^{2}=m_{e} / m_{i}$, and $M=U / a$ is the Alfvén Mach number based on the velocity of the plasma normal to the wave.

These equations are valid provided the $x$-component of the plasma velocity does not vanish, so that the particle trajectories do not loop back upon themselves. We now search for solutions which satisfy $u \rightarrow 1, E \rightarrow 0, h_{2}^{2}+h_{3}^{2} \rightarrow \sin ^{2} \beta$ as 
$x \rightarrow-\infty$. Clearly, we can without loss of generality replace the last condition by $h_{2} \rightarrow \sin \beta, h_{3} \rightarrow 0$. The cases of the transverse and longitudinal waves can be obtained by putting $\beta=\frac{1}{2} \pi$ and $\beta=0$, respectively. $\dagger$

\section{Reduction of the equations}

In the present problem it is convenient to follow the method of Montgomery (1959), who considered periodic waves of infinite extent rather than that previously employed by the author. We introduce a (dimensionless) time $t$ by

$$
d x / d t=u(x),
$$

and henceforth use $t$ as the independent variable. Differentiation with respect to $t$, which we denote by a dot, is differentiation following a particle.

It is easy to show, by adding (2.2) to (2.3) and using (2.4), that the equations possess three first integrals. These are

$$
\begin{gathered}
\gamma^{-1} v_{i}+\gamma v_{e}-\left(\gamma+\gamma^{-1}\right) M^{-2} h_{2} \cos \beta=\left(\gamma+\gamma^{-1}\right) \tan \beta\left(1-M^{-2} \cos ^{2} \beta\right) \\
\gamma^{-1} w_{i}+\gamma w_{e}-\left(\gamma+\gamma^{-1}\right) M^{-2} h_{3} \cos \beta=0 \\
u+\frac{1}{2} M^{-2}\left(h_{2}^{2}+h_{3}^{2}\right)=1+\frac{1}{2} M^{-2} \sin ^{2} \beta .
\end{gathered}
$$

The arbitrary constants have been fixed by the conditions at $x=-\infty$. These equations express the conservation of momentum.

Combining (3.2) and (3.3) with (2.4), we obtain the transverse velocities in terms of the magnetic field and its derivatives. Thus,

$$
\begin{aligned}
v_{i} & =h_{2} M^{-2} \cos \beta-\gamma \dot{h}_{3} M^{-1}+\tan \beta\left(1-M^{-2} \cos ^{2} \beta\right), \\
v_{e} & =h_{2} M^{-2} \cos \beta+\gamma^{-1} \dot{h}_{3} M^{-1}+\tan \beta\left(1-M^{-2} \cos ^{2} \beta\right), \\
w_{i} & =h_{3} M^{-2} \cos \beta+\gamma \dot{h}_{2} M^{-1}, \\
w_{e} & =h_{3} M^{-2} \cos \beta-\gamma^{-1} \dot{h}_{2} M^{-1} .
\end{aligned}
$$

We now substitute these expressions into (2.2) and (2.3) to obtain

$$
\begin{gathered}
\ddot{h}_{2}+\left(\gamma^{-1}-\gamma\right) M^{-1} \dot{h}_{3} \cos \beta-h_{2}\left(u-M^{-2} \cos ^{2} B\right)=-\sin \beta\left(1-M^{-2} \cos ^{2} \beta\right), \\
\ddot{h}_{3}-\left(\gamma^{-1}-\gamma\right) M^{-1} \dot{h}_{2} \cos \beta-h_{3}\left(u-M^{-2} \cos ^{2} \beta\right)=0 .
\end{gathered}
$$

Here, $u$ is given by (3.4) in terms of $h_{2}$ and $h_{3}$, and we are interested in solutions which satisfy $h_{2} \rightarrow \sin \beta, h_{3} \rightarrow 0$ as $t \rightarrow-\infty$, and which are such that $u$ is always positive. Once $h_{2}$ and $h_{3}$ are known, the other variables follow immediately.

Necessary but (and it is important to stress) not sufficient conditions for a solution to exist can be determined by investigating the form of the equations when linearized in $h_{2}-\sin \beta=\zeta_{2}$, say, and $h_{3}=\zeta_{3}$, say, i.e. near $t$ or $x=-\infty$. The linearized equations are

$$
\ddot{\zeta}_{2}+\lambda \dot{\zeta}_{3}-\mu \zeta_{2}=0, \quad \ddot{\zeta}_{3}-\lambda \dot{\zeta}_{2}-\nu \zeta_{3}=0
$$

where

$$
\lambda=\left(\gamma^{-1}-\gamma\right) M^{-1} \cos \beta, \quad \mu=1-M^{-2}, \quad \nu=1-M^{-2} \cos ^{2} \beta .
$$

$\dagger$ There is an apparent difficulty about $\beta=\frac{1}{2} \pi$, as with the present geometry it makes the velocity, in the plane of the wave, infinite. However, this trouble is not real, and is overcome by dropping the requirement that $E$ vanishes at infinity. The final equations ( 33 ) are in fact independent of the particular value of $E$ at $x=-\infty$, and we take $E=0$ simply for convenience. 
If we try solutions in which $\zeta_{2}$ and $\zeta_{3}$ are proportional to $e^{p t}$, we obtain the quadratic for $p^{2}$ :

$$
p^{2}+\mu \nu p^{-2}=\mu+\nu-\lambda^{2} .
$$

There are various cases depending on the relative magnitudes of $\mu, \nu$ and $\lambda$.

Case (i). $\nu>0>\mu$, or $\cos \beta<M<1$. The quadratic for $p^{2}$ then has two real roots, one positive and the other negative. The negative root gives pure imaginary values for $p$ which cannot satisfy the condition that the solution vanishes as $t \rightarrow-\infty$. Hence, in this case there is at most a unique solution describing the waves. (The arbitrary constant in the solution can be absorbed into the position of the origin of $t$ and, equivalently, $x$.) It will be a valid solution if it satisfies $u>0$ everywhere, but this is a very much more difficult question which we shall leave for the moment.

Case (ii). $\nu>\mu>0$, or $M>1$. The quadratic for $p^{2}$ has two positive roots if $\mu+\nu-\lambda^{2}>2 \sqrt{ }(\mu \nu)$, and two complex roots if $2 \sqrt{ }(\mu \nu)>\mu+\nu-\lambda^{2}>-2 \sqrt{ }(\mu \nu)$. In either case, there are two values of $p$ with positive real part, and there may be therefore an infinite number of solutions depending upon the relative values of the coefficients of the two exponential terms.

On substituting for $\mu, \nu$ and $\lambda$, we find that there are two cases according as

or

$$
\begin{gathered}
\cos ^{2} \beta<\left\{1+\left(\gamma^{-1}-\gamma\right)^{2}\right\}^{-1}, \quad \text { i.e. } \quad \tan \beta>\gamma^{-1}-\gamma \\
\cos ^{2} \beta>\left\{1+\left(\gamma^{-1}-\gamma\right)^{2}\right\}^{-1}
\end{gathered}
$$

In the former case, $p^{2}$ has two positive roots for $1<M<M_{1}$, and two complex roots for $M>M_{1}$. In the latter case, $p^{2}$ has two complex roots provided $M>M_{1}$ and two negative roots for $M<M_{1}$. Here

$$
M_{1}^{2}=1+\frac{\left[\cos ^{2} \beta\left\{1+\left(\gamma^{-1}-\gamma\right)^{2}\right\}-1\right]^{2}}{4\left(\gamma^{-1}-\gamma\right)^{2} \cos ^{2} \beta} .
$$

(These results follow without difficulty from a consideration of the roots of $\mu+\nu-\lambda^{2}= \pm 2 \sqrt{ }(\mu \nu)$. These changes in the nature of the solutions for particular values of $\beta$ and $M$ are puzzling, and the author has not seen any obvious physical interpretation.)

Case (iii). $\mu=\nu>0$, or $\beta=0, M>1$. The values of $p$ are complex with nonzero real part if $\mu>\lambda^{2}$, i.e. $M>\frac{1}{2}\left(\gamma^{-1}+\gamma\right)$. Otherwise they are pure imaginary. There are infinitely many solutions, but they are all essentially the same and can be obtained from one another by rotating about the normal to the wave plane.

Case (iv). $\mu=0$ or $M=1$. One of the roots of the quadratic for $p^{2}$ is zero, and the corresponding solution must be zero. The other root is positive if $(3.13 a)$ is satisfied, in which case there is a unique solution; and is negative if $(3.13 b)$ is satisfied in which case there is no non-trivial solution.

Case (v). $0>\nu>\mu$, or $M<\cos \beta$. The quadratic has two negative roots, so the only solution is the trivial one in which all variables are constant.

To summarize the conclusions of this section, we have obtained the basic equations for finite amplitude waves and determined the conditions for solutions to exist which initially grow exponentially from a uniform state. These are 
necessary, but not sufficient, for the solutions must be bounded for all $t$ and must also satisfy $u>0$ if they are going to represent finite amplitude waves with a real physical meaning. For this purpose it is useful to notice that the basic equations (3.9) and (3.10) describe the motion of a particle in a uniformly rotating field of force, which is the superposition of a central force and a constant acceleration, and we shall for the sake of clarity discuss the equations in terms of this analogy. This is, in fact, a particular form of the restricted three-body problem.

\section{Analogy with the motion of a particle in a uniformly rotating field of force}

If we write $X=h_{2}, Y=h_{3}$, then, from (3.9) and (3.10), $X, Y$ are the co-ordinates of a particle of unit mass moving in a plane under a conservative field of force with potential

$$
V=\frac{1}{8} M^{-2}\left(X^{2}+Y^{2}-\sin ^{2} \beta\right)^{2}-\frac{1}{2}\left(1-M^{-2} \cos ^{2} \beta\right)\left\{(X-\sin \beta)^{2}+Y^{2}\right\},
$$

and subject to a Coriolis force of amount $\lambda$ times its velocity, where

$$
\lambda=\left(\gamma^{-1}-\gamma\right) M^{-1} \cos \beta .
$$

The point $X=\sin \beta, Y=0$ is a point of equilibrium (the arbitrary constant in $V$ has been chosen so that $V=0$ there) and corresponds to the uniform state at upstream infinity.

The motion has an energy integral

$$
\frac{1}{2}\left(\dot{X}^{2}+\dot{Y}^{2}\right)+V=0,
$$

where the total energy is zero for particles at the equilibrium point at $t=-\infty$. The particle orbit is confined to the region $V<0$, and it is clear from inspection that this region is bounded and of finite extent. Hence, all solutions of the equations are bounded.

The curve $V=0$ is sketched in figure 2. There are three cases, corresponding with cases (i), (ii) and (iii) of the previous section. $A$ is the point of equilibrium, and $B$ and $C$ are the other intersections of $V=0$ with the $X$-axis. The curves are symmetrical about this axis.

In case (i), there is a unique orbit starting from $A$. In case (ii), there are infinitely many orbits or none, depending upon the values of $\beta$ and $M$ [see (3.13) and (3.14)]. In case (iii) (longitudinal waves) the solution is essentially unique if it exists.

The number of exact solutions which have so far been obtained is very limited. Case (iii) has been solved completely (Saffman 1961). Solutions for case (ii) can be obtained if $\lambda=0$ (zero Coriolis force), which occurs if $\beta=\frac{1}{2} \pi$ (transverse waves) or $\gamma=1$ (i.e. $m_{i}=m_{e}$ ). In these, $Y \equiv 0$ and the orbit is from $A$ to $B$ and back again. For the case $\beta=\frac{1}{2} \pi$, these solutions were found by Adlam \& Allen (1958) and Davis et al. (1958); the orbit from $A$ to $C$ and back again, which entails a reversal in the magnetic field, is not a possible solution because it violates, as we shall see later, the condition $u>0$.

It may be mentioned here in passing that the equations governing the motion of the particle, (3.9) and (3.10) with $X=h_{2}$ and $Y=h_{3}$, are invariant under the transformation $t \rightarrow-t^{\prime}, X \rightarrow X^{\prime}, Y \rightarrow-Y^{\prime}$. Hence, symmetrical solutions exist 


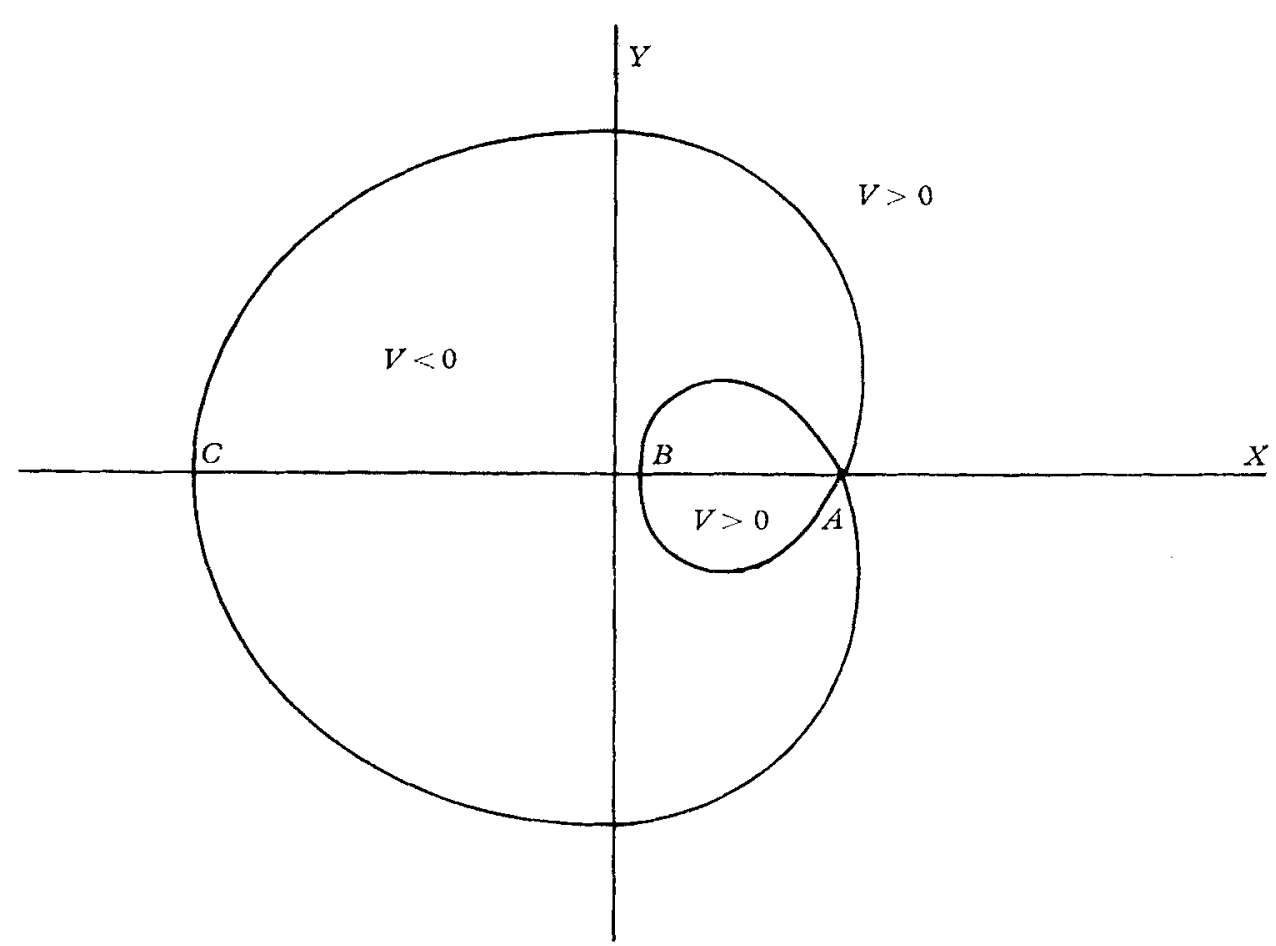

(i) $\cos \beta<M<1$
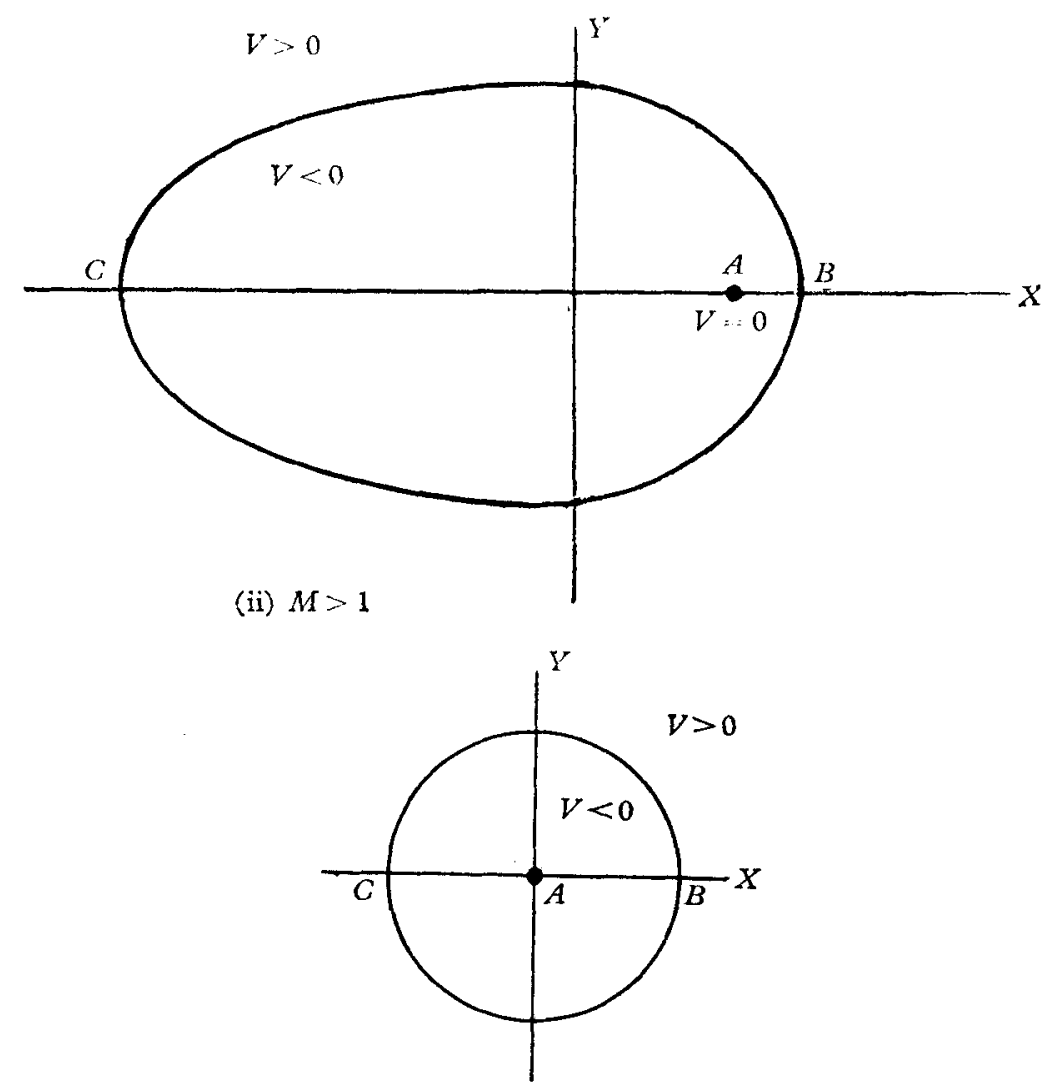

(iii) $\beta=0, M>1$

Frgure 2. Sketch of the accessible portions of the $(X, Y)$-plane for cases (i), (ii) and (iii). All orbits start from the point $A$. 
if and only if $\dot{X}$ and $Y$ vanish together for some value of $t$. In terms of the plasma waves, the condition for symmetrical waves is that a plane should exist on which $d h_{2} / d x$ and $h_{3}$ vanish together. The exact solutions just mentioned are symmetrical waves.

\section{Oblique solitary waves in a plasma with mass ratio unity}

For the sake of completeness, we shall describe briefly the oblique solitary waves in a plasma with $m_{i}=m_{e}$, since they (although perhaps mainly of academic interest) have not apparently been reported before. The equations admit of a solution for all $M>1$ with $Y=h_{3}=0$; and (4.2) becomes

$$
\frac{1}{2} \dot{X}^{2}=\frac{1}{8} M^{-2}(X-\sin \beta)^{2}\left\{4\left(M^{2}-1\right)-4 \sin \beta(X-\sin \beta)-(X-\sin \beta)^{2}\right\} .
$$

Hence,

$$
X=\sin \beta+\left[\frac{1}{2} \frac{\sin \beta}{M^{2}-1} \pm\left\{\frac{1}{4} \frac{\sin ^{2} \beta}{\left(M^{2}-1\right)^{2}}+\frac{1}{4\left(M^{2}-1\right)}\right\}^{\frac{1}{2}} \cosh t \sqrt{\frac{M^{2}-1}{M}}\right]^{-1} .
$$

The plus sign corresponds to the orbit from $A$ to $B$, and the minus sign with that from $A$ to $C$. These solutions are symmetrical about $t=0$ and therefore describe solitary waves.

We must now consider the value of $u$, to determine whether the solution has physical validity. By (3.4),

$$
u=1+\frac{1}{2} M^{-2} \sin ^{2} \beta-\frac{1}{2} M^{-2} X^{2}
$$

and the minimum value of $u$ occurs at $B$ and $C$, which have co-ordinates

$$
\begin{aligned}
& X_{B}=-\sin \beta+2\left(M^{2}-\cos ^{2} \beta\right)^{\frac{1}{2}}, \\
& X_{C}=-\sin \beta-2\left(M^{2}-\cos ^{2} \beta\right)^{\frac{1}{2}} .
\end{aligned}
$$

Then

$$
\begin{aligned}
& u(B)=-1+2 M^{-2} \cos ^{2} \beta+2 M^{-2} \sin \beta\left(M^{2}-\cos ^{2} \beta\right)^{\frac{1}{2}}, \\
& u(C)=-1+2 M^{-2} \cos ^{2} \beta-2 M^{-2} \sin \beta\left(M^{2}-\cos ^{2} \beta\right)^{\frac{1}{2}} .
\end{aligned}
$$

On the other hand, $u(C)<0$ for all $M(>1)$ if

$$
\cos 2 \beta<\frac{1}{2} \text {, i.e. } \beta>\frac{1}{6} \pi \text {. }
$$

But if $\beta<\frac{1}{6} \pi, u(C)>0$ for

$$
M^{2}<2-2 \sin \beta
$$

Thus, if $\beta>\frac{1}{6} \pi$, only the solution corresponding to the path from $A$ to $B$, which means that the magnetic field increases, is physically relevant. But if $\beta<\frac{1}{6} \pi$, the other solution from $A$ to $C$ in which the direction of the transverse magnetic field reverses may also be valid, and there is more than one possible solitary wave. The values of all the other variables can be found from (5.2) and the equations of $\S 3$.

We note now for future use that the results (5.3) to (5.9) hold generally for case (ii) $(M>1)$ whatever the value of the Coriolis effect $\lambda$. Moreover, if $u(C)>0$, then $u>0$ everywhere in $V<0$. This completes the discussion of exact solutions, and we now consider the general case. 


\section{General properties of the orbits}

The solutions of the equations are repesented by orbits which leave the point $A$ of figure 2 and are contained within the region $V<0$. The orbits can be of two types, closed or open. By a closed orbit we mean one that returns to $A$ as $t \rightarrow+\infty$, and by an open one we mean an orbit that never returns to $A$ but wanders indefinitely through the permissible region of the plane. The closed orbits would give solitary waves, with conditions the same at upstream and downstream infinity. (There is no a priori reason for saying that the structure of the solitary waves is symmetrical.) The open orbits would give a motion in which the variables oscillate indefinitely as $t \rightarrow+\infty$, which is what we have called a quasi-shock. A true shock would require the existence of another equilibrium point with $V=0$ to which the particle would tend as $t \rightarrow+\infty$, and such a point is easily shown not to exist. Unfortunately, it is a very difficult question to determine which orbits are open or closed. However, this type of problem has been examined by workers in classical dynamics (see, for example, Birkhoff 1927; Khinchin 1947) and it is a very plausible assumption that the quasi-ergodic theorem applies to the dynamical system we are considering.

The consequence of making this plausible assumption is that closed orbits may exist but there are not more than an enumerable infinity of them. The open orbits, if they exist, are such that the trajectories in four-dimensional phase space $(\dot{X}, \dot{Y}, X, Y)$ lie on the three-dimensional manifold $\dot{X}^{2}+\dot{Y}^{2}+2 V=0$ and go infinitely near any point of it an infinite number of times, and their density in phase space is asymptotically uniform. Thus an average over a sufficiently large time is equivalent to an average over the accessible region of phase space.

Consider now our three cases. For case (i), there is a unique solution which may be a solitary wave or a quasi-shock. For case (ii), the number of orbits is a nonenumerable infinity (since an orbit is determined by the ratio of two arbitrary parameters), and there probably are solitary waves, but there will be quasi-shocks. For case (iii), there is essentially one solution which is a solitary wave. [The longitudinal waves of case (iii) are fundamentally different from cases (i) and (ii) $\left(0<\beta \leqslant \frac{1}{2} \pi\right)$ and are included here for the sake of completeness. The reason is that when $\beta=0$ the equations have another integral because the angular momentum (relative to a certain frame) is conserved. The motion therefore has only one degree of freedom as opposed to cases (i) and (ii) where there are two degrees of freedom.] Note that by the quasi-ergodic theorem, time averages following a particle are the same for each quasi-shock (for given $M$ and $\beta$, of course).

It remains now to consider which, if any, of these possible orbits will satisfy $u>0$, which is necessary if our equations are to be a valid description of the flow. We have from (3.4) that $u>0$ if

$$
X^{2}+Y^{2}<2 M^{2}+\sin ^{2} \beta
$$

i.e. provided the particle remains inside a circle of radius $\left(2 M^{2}+\sin ^{2} \beta\right)^{\frac{1}{2}}$. This circle contains the point $A$, so all solitary waves which stay sufficiently close to $A$ will be possible. The necessary conditions for the case of the waves in $\$ 5$ and the longitudinal waves, we already know. More than this we cannot find for the solitary waves. 
However, the problem is easily solved for the quasi-shocks, since then the orbits go almost everywhere, and the condition reduces to $u(C)>0$ since $C$ is the point farthest from the origin in the region $V<0$.

For case (i), with $\cos \beta<M<1$, it follows from (5.6) that $u(C)>0$ for $M^{2}<2-2 \sin \beta$ which is (5.9). (Note that $2-2 \sin \beta>\cos ^{2} \beta$.) Thus for case (i), whatever the solution, i.e. whether or not it is a solitary wave or a quasi-shock, it is physically significent if the Alfvén Mach number lies between $\cos \beta$ and the smaller of $(2-2 \sin \beta)^{\frac{1}{2}}$ and 1 .

Case (ii) is more difficult. As shown at the end of $\S 5, u(C)>0$ only if $\beta<\frac{1}{6} \pi$ and $M^{2}<2-2 \sin \beta$. However, the conditions expressed by (3.13) and (3.14) for the orbit to leave the vicinity of $A$ must also be satisfied, and these depend upon the value of the mass ratio of the ions and electrons. If $\gamma^{2}=m_{e} / m_{i}>(7-\sqrt{13}) / 6$, then (3.13 $a$ ) is satisfied, and quasi-shocks exist, for the range of values of $\beta$ which satisfy (3.13a). For smaller $\beta$, they exist only if

$$
(2-2 \sin \beta)^{\frac{1}{2}}>M>M_{1} \text {. }
$$

If $\gamma^{2}<(7-\sqrt{ } 13) / 6$, which is the case for Hydrogen, they exist only if $(6.2)$ is satisfied (with $\beta<\frac{1}{6} \pi$ ). We can show that it is impossible to satisfy (6.2) if $\gamma<\sqrt{ } 2-1$, which means that quasi-shocks with $M>1$ do not exist for Hydrogen, although they may for a plasma with mass-ratio closer to unity.

We shall now calculate in the next section the mean properties of the plasma behind a quasi-shock for case (i). It should be noted, incidentally, that if the electric field ahead of the wave is taken to be zero, the total velocity of the plasma is $U \sec \beta$, and the Alfvén Mach number based on this velocity, $M^{*}$ say, is equal to $M \sec \beta$. Thus, for quasi-shocks in case (i),

$$
\begin{array}{cl}
1<M^{*}<\sec \beta \quad \text { if } & 0<\beta<\frac{1}{6} \pi, \\
1<M^{*}<\{2 /(1+\sin \beta)\}^{\frac{1}{2}} & \text { if } \quad \frac{1}{6} \pi<\beta<\frac{1}{2} \pi .
\end{array}
$$

The maximum permissible value of $M^{*}$ occurs for $\beta=\frac{1}{6} \pi$ and is $2 / \sqrt{ } 3$. In any case, $M^{*}$ is greater than one, so we are not really finding shock-like motions with Alfvén Mach number less than one.

\section{Mean values behind a quasi-shock}

It seems plausible that in case (i), where there is a unique solution of the equations and of the conditions at $t=-\infty$, that it will depend upon the particular values of $\gamma, M$ and $\beta$ whether the wave is a solitary wave or a quasi-shock. That is to say, it is not likely that the solution will always be a solitary wave. For suppose one orbit is closed, and we change the force field slightly by putting a small bump in the path. Then the particle is deflected and will wander around. It seems unlikely that subsequent passages across the bump can have the effect of putting it back on to an orbit which returns exactly to the starting-point $A$. Thus, we expect the open orbit to be the rule, and the closed orbit to be the exception. A further supporting argument for considering quasi-shocks will be given in $\S 8$.

Now we cannot calculate the detailed properties of the quasi-shock, but by the quasi-ergodic theorem we can calculate the mean values following a particle, these being the mean values over the phase space, and we can deduce space 
averages for the plasma waves. This is, of course, entirely sufficient for our purposes, for such mean values are all that could be observed.

The trajectory in phase space of the particle in the mechanical analogue lies on a hyper-surface of constant energy in four-dimensional phase space. For given $\dot{X}$ and $\dot{Y}$, the surfaces of constant energy are circles in the $X, Y$-plane. The appropriate way of taking phase averages of a function of the phase-space co-ordinates is to integrate it over the volume of phase space between the hyper-surface of constant energy and a neighbouring one, divide the integral by the volume of integration to obtain an average, and take the limit as the distance between the hyper-surfaces tends to zero. (For a full discussion of the method of taking averages, see Khinchin 1947.) It follows that the phase average, which will be denoted by angle brackets, of a function $f(X, Y)$ independent of $\dot{X}$ and $\dot{Y}$, is

$$
\langle f\rangle=\frac{\iint f d X d Y}{\iint d X d Y}, \dagger
$$

the integration being over the region $V<0$ of the $X, Y$-plane (see figure 2(i)), Phase averages of functions involving the velocity in the orbit are

$$
\langle\dot{X} f\rangle=\langle\dot{Y} f\rangle=0,\left\langle\dot{X}^{2} f\right\rangle=\left\langle\dot{Y}^{2} f\right\rangle=-\langle V f\rangle \text {, etc. }
$$

In terms of the plasma waves, these phase averages give the asymptotic time averages behind the wave front following the motion of a particle (ion or electron). These will be the appropriate ones for the average energy and momentum per particle. However, the particles are moving with variable velocity $u$ in the $x$-direction; and the time averages will not be simply proportional to the space averages over $x$, which will be more appropriate in the case of the magnetic field. The velocity $u$ is given by (3.4) in terms of $X$ and $Y$, and the relation between space averages and time averages is (an over bar denoting space averages)

$$
\bar{f}=\langle f u\rangle \mid\langle u\rangle \text {. }
$$

For the physical quantities of most interest, i.e. the velocities and the magnetic field, $f$ is a polynomial in $X$ and $Y$. The double integral can then be evaluated by using polar co-ordinates centred on $A$ (see figure $2(\mathrm{i})$ ), and by first integrating with respect to the distance from $A$. In these co-ordinates, defined by

$$
\sin \beta-X=r \cos \theta, \quad Y=r \sin \theta,
$$

the bounding curves $V=0$ have the equations

$$
r=2 \cos \theta \sin \beta \pm 2\left(M^{2}-\cos ^{2} \beta\right)^{\frac{1}{2}} .
$$

The integrations are now straightforward, but long and tedious, and the final expressions are not at all simple. They are indeed too complicated for general use, and it was not thought worth while working them out in full. For our present purpose of determining the general features of quasi-shocks, it is sufficient to give the results for the two limiting cases at the ends of the range for which case (i) applies: these are case $(a) 0<M-\cos \beta \ll 1$, and case $(b) 0<1-M \ll 1$ (note that case $(b)$ requires $\left.\beta<\frac{1}{6} \pi\right)$.

† I am indebted to Dr F. D. Kahn for pointing out an error here in an earlier draft and for giving the correct method of taking phase averages. 
Case (a). $M \doteqdot \cos \beta$. We find that

$$
\begin{gathered}
\left\langle h_{2}\right\rangle=\langle X\rangle \doteqdot-\frac{1}{3} \sin \beta,\left\langle h_{3}\right\rangle=\langle Y\rangle=0 . \\
\left\langle h_{2}^{2}\right\rangle \div \frac{7}{15} \sin ^{2} \beta,\left\langle h_{3}^{2}\right\rangle \doteqdot \frac{8}{15} \sin ^{2} \beta .
\end{gathered}
$$

Then from (3.5) to (3.8), we have that

$$
\left\langle v_{i}\right\rangle=\left\langle v_{e}\right\rangle \doteqdot-\frac{1}{3} \tan \beta,\left\langle w_{i}\right\rangle=\left\langle w_{e}\right\rangle=0 .
$$

Also, $u \doteqdot 1$, so that to this order of approximation, space and time averages are the same. The mean-square variations of the velocities are

$$
\left.\begin{array}{rl}
\left\langle(u-\langle u\rangle)^{2}\right\rangle & =0, \\
\left\langle\left(v_{i}-\left\langle v_{i}\right\rangle\right)^{2}\right\rangle & \doteqdot\left\langle\left(v_{e}-\left\langle v_{e}\right\rangle\right)^{2}\right\rangle \doteqdot \frac{16}{45} \tan ^{2} \beta, \\
\left\langle\left(w_{i}-\left\langle w_{i}\right\rangle\right)^{2}\right\rangle & \doteqdot\left\langle\left(w_{e}-\left\langle w_{e}\right\rangle\right)^{2}\right\rangle \doteqdot \frac{8}{15} \tan ^{2} \beta .
\end{array}\right\}
$$

Thus, for $M$ sufficiently close to $\cos \beta$, there is no difference in the mean properties of the ion and electron velocities.

If we interpret these mean-square variations of velocity as a temperature, they imply that the temperature behind the quasi-shock is non-isotropic, with zero temperature in the direction of the wave normal and maximum temperature in the direction in which there is no mean magnetic field. (It is to be remembered that the above velocities are non-dimensional.) Owing to the greater mass of the ions, most of the 'heat' is carried by the ions. The orientation of the mean flow and magnetic fields is shown in figure $3(a)$. The mean flow is turned back through the angle $\beta+\beta^{\prime}$, where $\tan \beta^{\prime}=\frac{1}{3} \tan \beta$.

Case (b). $M \doteqdot 1\left(\beta<\frac{1}{6} \pi\right)$. We find that:

$$
\left.\begin{array}{rl}
\langle u\rangle & \doteqdot 1-\frac{5}{4} \sin ^{2} \beta, \\
\left\langle v_{i}\right\rangle & =\left\langle v_{e}\right\rangle=-\frac{2}{3} \sin \beta \cos \beta\left(1-\frac{3}{2} \tan ^{2} \beta\right), \\
\left\langle w_{i}\right\rangle & =\left\langle w_{e}\right\rangle=0 .
\end{array}\right\}
$$

The time average of the magnetic field is

$$
\left\langle h_{2}\right\rangle \div-\frac{2}{3} \sin \beta,\left\langle h_{3}\right\rangle=0 .
$$

The space average of the magnetic field is different and is

$$
\bar{h}_{2} \doteqdot-\frac{2}{3} \sin \beta\left(1-\frac{5}{2} \sin ^{2} \beta\right), \quad \bar{h}_{3}=0 .
$$

It is interesting that the mean direction of the particles is not parallel to either of the magnetic field averages (see figure $3(b)$ ).

The mean-square time averages of the particle velocities are:

$$
\left.\begin{array}{l}
\left\langle u^{2}\right\rangle \doteqdot 1+\frac{1}{6} \sin ^{2} \beta+\frac{35}{12} \sin ^{4} \beta, \\
\left\langle v_{i}^{2}\right\rangle \doteqdot \frac{7}{4} \sin ^{2} \beta \cos ^{2} \beta+\sin ^{4} \beta \tan ^{2} \beta-\frac{4}{3} \sin ^{4} \beta+\frac{7}{24} \gamma^{2} \sin ^{4} \beta, \\
\left\langle v_{e}^{2}\right\rangle \doteqdot \frac{7}{4} \sin ^{2} \beta \cos ^{2} \beta+\sin ^{4} \beta \tan ^{2} \beta-\frac{4}{3} \sin ^{4} \beta+\frac{7}{24} \gamma^{-2} \sin ^{4} \beta, \\
\left\langle w_{i}^{2}\right\rangle \doteqdot \frac{7}{4} \sin ^{2} \beta \cos ^{2} \beta+\frac{7}{24} \gamma^{2} \sin ^{4} \beta, \\
\left\langle w_{e}^{2}\right\rangle \doteqdot \frac{7}{4} \sin ^{2} \beta \cos ^{2} \beta+\frac{7}{24} \gamma^{-2} \sin ^{4} \beta .
\end{array}\right\}
$$


The expressions for cases $(a)$ and $(b)$ are generally of the same order of magnitude. The exception is the velocity fluctuations of the electrons. Remembering that $\gamma^{2}=m_{e} / m_{i}$ is a small quantity for a real plasma, we see that the transverse fluctuations of the electron velocity are $\gamma^{-1}$ times larger than the other velocities. It follows that the 'temperature' of the transverse electron motion is comparable
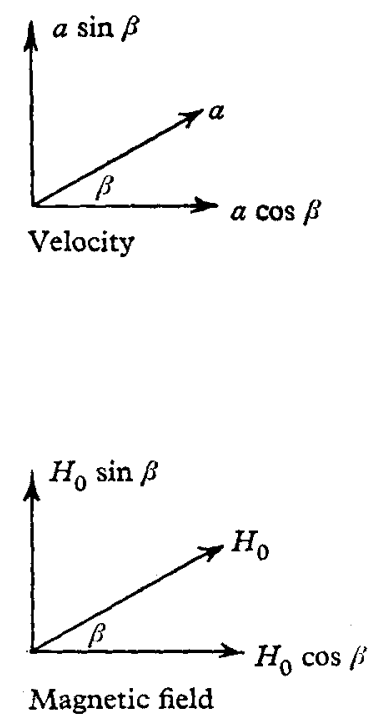

(a) $M \doteqdot \cos \beta$
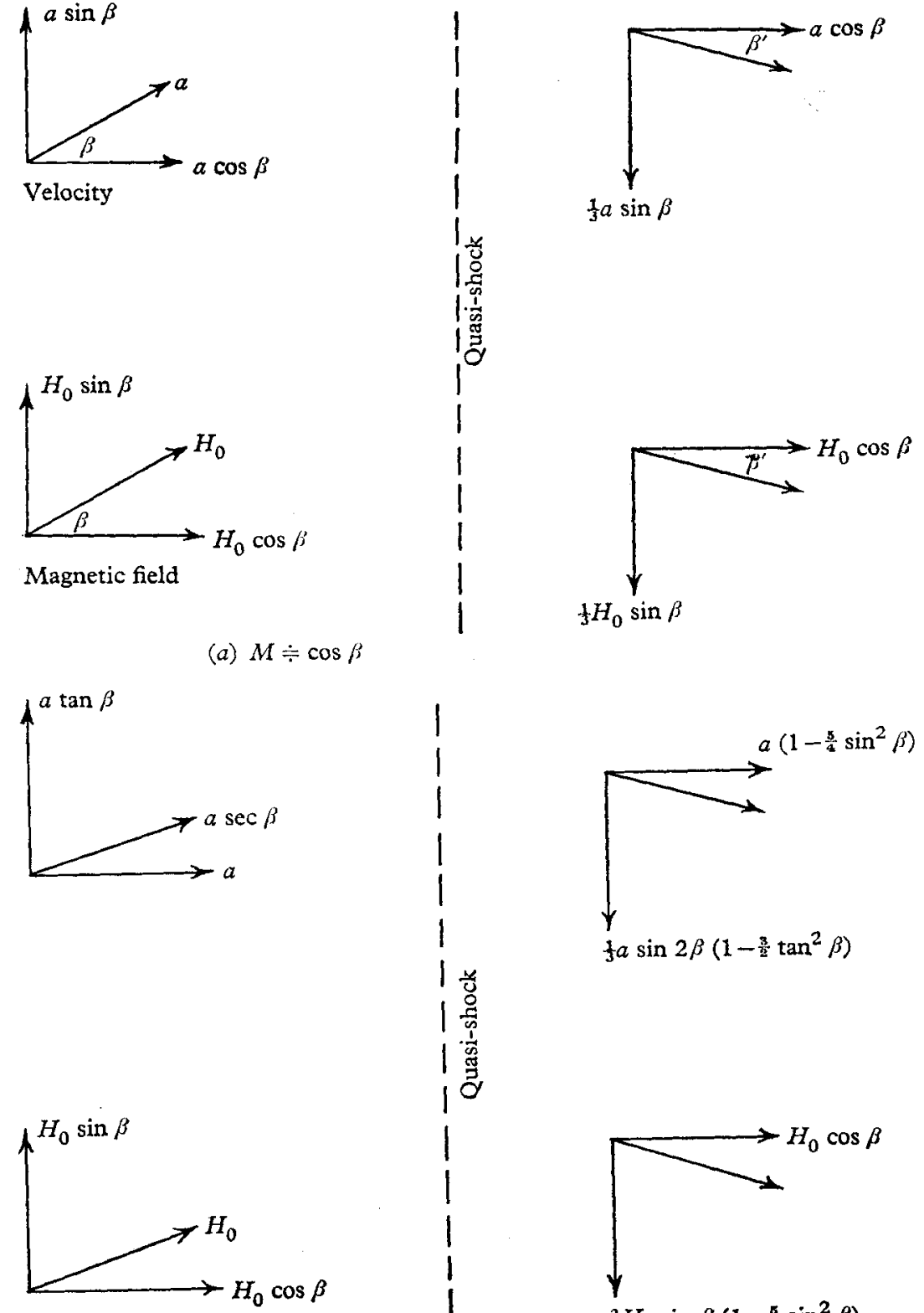

(b) $M \div 1$

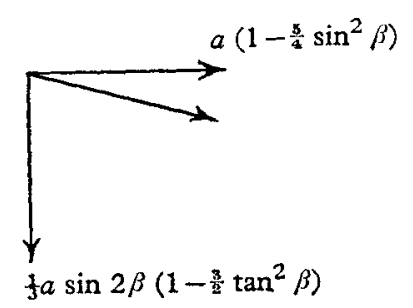

1 \%
$1 \frac{0}{0}$
10
1
1
0
0
1
1
1
1

Figure 3. The mean flow behind a quasi-shock, for the cases $(a)$ when the upstream velocity is just greater than the Alfvén velocity $a$, and (b) when it is just less than $a$ sec $\beta$. 
with that of the ions. This effect will disappear only when $M$ is close to $\cos \beta$. The temperature of the longitudinal velocity fluctuations (i.e. in the $x$-direction) is much greater for the ions than for the electrons.

The quasi-shocks are essentially irreversible motions which transform a uniform state into an irregularly varying one, and provide an example of a dissipation mechanism in a cold collision-free plasma, which does not invoke collisions, electrostatic oscillations, or require the plasma ahead of the wave to be warm. It is to be emphasized that there is no spread of velocity at a fixed point of space, so that strictly speaking the plasma remains cold on passing through a quasi-shock, but an observer on a scale large compared with the ion gyro-radius would appear to see random motions superposed on a uniform state, i.e. the plasma would look warm.

An estimate of the width of the quasi-shock or more precisely the transition region can be obtained by considering the rate at which the disturbance to the uniform state grows, as was done in $\$ 3$. In dimensional quantities, we can define the width as $d=l / p$, where $p$ is given by (3.12) and $l$ is the geometric mean of the ion and electron gyro-radii. For hydrogen, $\gamma$ is small and

$$
\frac{l}{p} \doteqdot \frac{\gamma^{-1} M \cos \beta}{\left[\left(M^{2}-\cos ^{2} \beta\right)\left(1-M^{2}\right)\right]^{\frac{1}{2}}} \text {. }
$$

Thus $d$ is of the order of the ion gyro-radius.

The features of the oscillation behind the transition region are difficult to establish with certainty. From an inspection of (3.9) and (3.10), it can be seen that the largest term in each equation is the second or 'Coriolis' term involving $\lambda$ (see 3.11). In general, this will balance either the first term in each equation, giving fast oscillations with $d / d t \propto \lambda$ (i.e. at the electron gyro-frequency), or the last term giving slow oscillations with $d / d t \propto \lambda^{-1}$ (i.e. at the ion gyro-frequency). The fast ones must be of small amplitude $O\left(\lambda^{-1}\right)$ in the magnetic field, in order that the 'energy' integral (4.2) be satisfied; and it follows from (3.5) to (3.8) that they mainly affect the electrons, giving the electrons energies comparable with that of the ions in the slow oscillations. Thus the general picture seems to be one of velocity oscillations of the ions and electrons with comparable amplitude at the ion gyro-frequency, the energy being carried by the ions, on which are superposed oscillations of the electrons at the electron gyro-frequency with a comparable amount of energy.

The relation of the present theory to experiment is limited of course by the fact that a real plasma would have a non-zero temperature, but it is not clear at present. exactly how this would change the waves described here. Amongst other things, Landau damping in a hot plasma would possibly become important and have to be taken into account, providing an extra dissipative process.

\section{Stability of the waves}

We have seen that the waves can be represented by the orbits of a particle in a uniformly rotating field of force, and that there are two types of orbit, open or closed, if $\beta>0$. Now the effect of small disturbances will be to introduce small perturbations on the right-hand side of the equations of motion (3.9) and (3.10) 
which will cause the particle to move slowly from one orbit to a neighbouring orbit in phase space. Now the number of closed orbits is enumerable, and there are infinitely more open orbits. Thus even if the conditions at $t=-\infty$ are such that the particle is initially on a closed orbit, any small disturbance (except those of a very special kind) will put it on to an open orbit, and a disturbance to a particle on an open orbit will in general put it on to another open orbit. Thus the closed orbits are unstable. The mean properties of the open orbits are all the same, by the quasi-ergodic hypothesis, so even though an individual open orbit is unstable, the mean properties of particles on open orbits are stable in the sense that they are unaffected by small disturbances.

In terms of the waves, this means that the solitary-wave solutions are unstable and very much the exception, whereas the quasi-shocks are stable in mean, and will be the rule. (This is why the analysis of $\S 7$ is physically significant, even if the exact solution is a solitary wave.) In particular, the transverse solitary waves $\left(\beta=\frac{1}{2} \pi\right)$ are unstable and would not be expected to occur or be physically significant. The difficulty now is that quasi-shocks cannot be constructed which satisfy the condition $u>0$ when $M>1$, unless $\beta<\frac{1}{6} \pi$ and the mass ratio is sufficiently close to unity, which rules out Hydrogen. Thus the only physically significant solutions, which satisfy the condition that the ion and electron paths are not looped, are the quasi-shocks of $\S 7$ and the longitudinal solitary waves (Saffman 1961). For these latter waves correspond to a dynamical system with one degree of freedom, and small disturbances will only perturb or distort the orbit and not change its character.

It should be noted that the stability concept employed here is different from that usually understood in fluid mechanics, but resembles that sometimes employed in the theory of differential equations. We are not considering whether a small disturbance of the steady basic flow will grow or decay with time, but rather the question whether the steady solution can exist in a slightly perturbed form if steady external disturbances are present. The boundary conditions at $x=-\infty$ (the uniform state) are supposed fixed. Then the introduction of a small steady external disturbance does not affect the solution at upstream infinity, but in general has considerable effect on the solution at downstream infinity, and it is in this sense that the solitary-wave solutions with $\beta \neq 0$ are unstable. An individual quasi-shock is likewise unstable, but since they all have, in general, the same average properties, we can say that they are stable in mean.

\section{REFERENCES}

Adlam, J. H. \& Alten, J. E. 1958 Phil. Mag. 3, 448.

BIRKrorf, G. 1927 Theory of Dynamical Systems. New York: American Mathematical Society.

Davts, L., Lüst, R. \& SCHLÜTER, A. $1958 Z$. Naturforsch. $13 a, 916$.

Kmrлснт, A. I. 1947 Mathematical Foundations of Statistical Mechanics. New York: Dover.

Montgomery, D. 1959 Phys. Fluids, 2, 585.

Safrman, P. G. $1961 J$. Fluid Mech. 11, 16. 